\title{
Análisis de la eficiencia productiva del cultivo de alfalfa mediante regresión logística de datos categóricos en el Distrito de Riego 05-Delicias, Chihuahua, México \\ Analysis of productive efficiency of alfalfa cultivation by logistic regression of categorical data in the Irrigation District 05-Delicias, Chihuahua, Mexico
}

Octavio Villalobos Cano ${ }^{1}$, Esteban Sánchez Chávez ${ }^{3}$, Carlos Raúl Morales Nieto², Mario Edgar Esparza Vela ${ }^{2}$, Eduardo Santellano Estrada ${ }^{2}$

Palabras Clave: Medicago sativa L.; alfalfa; eficiencia productive; factores productivos Keywords: Medicago sativa L.; alfalfa; productive efficiency; productive factors

Recepción: 23-01-2018 / Aceptación: 10-04-2018

\section{Resumen}

Introducción: La alfalfa es la leguminosa más utilizada en la alimentación del ganado a nivel mundial, su alto valor nutritivo, principalmente por su contenido de proteína, lo hacen un ingrediente atractivo para su inclusión en la dieta. El Estado de Chihuahua es el principal productor de alfalfa en México y el Distrito de Riego 05-Delicias ocupa el segundo lugar en la producción de alfalfa de los distritos de riego en el país, con el 18\% la producción, pues este cultivo llega a cubrir casi la mitad de su superficie. El objetivo de este trabajo fue determinar el impacto de cuatro factores de producción sobre la eficiencia productiva del cultivo y generar información de utilidad para la toma de decisiones de productores y autoridades.

Método: En este estudio se seleccionaron cuatro variables predictoras (factores de producción) en escala dicotómica: Tenencia de la tierra, Propiedad del predio, Maquinaria especializada y Riego tecnificado, para determinar su efecto sobre la eficiencia productiva en el cultivo de alfalfa como variable de respuesta. Para determinar el mejor ajuste del modelo se recurrió al método de selección de variables por pasos hacia atrás (backward), los datos se analizaron bajo un modelo de Regresión Logística utilizando el procedimiento CATMOD del paquete SAS 9.4 y mediante contrastes para la determinación de los efectos de los factores en las interacciones significativas.

Resultados: Se encontró efecto significativo de la triple interacción Propiedad-Maquinaria especializada-Riego tecnificado, así como, en general, de los efectos principales Maquinaria especializada y Riego tecnificado. Al determinar, mediante contrastes, los efectos de las variables

\footnotetext{
${ }^{1}$ Estudiante de Posgrado del Programa de Doctorado en Recursos Naturales de la Facultad de Zootecnia y Ecología de la Universidad Autónoma de Chihuahua

${ }^{2}$ Profesor Investigador de la Facultad de Zootecnia y Ecología de la Universidad Autónoma de Chihuahua. E-mail: esantellano@uach.mx

${ }^{3}$ Profesor investigador del Centro de Investigación en Alimentación y Desarrollo A.C.

(C) Universidad De La Salle Bajío (México)
} 
dentro de la triple interacción, sobresalió el impacto potencial que produce la existencia de Maquinaria especializada-Riego tecnificado en conjunto, cuando se cuenta con la propiedad del predio con un valor tasa de momios de 16 (número de veces en que se incrementa la probabilidad potencial de alcanzar la eficiencia productiva con estos factores de producción); seguido del efecto de la Maquinaria especializada cuando se tiene la Propiedad, con una tasa de momios de 13.56; y el efecto del Riego tecnificado, cuando se tiene la Propiedad del predio con una tasa de momios de 8.0.

Discusión o Conclusión: La eficiencia productiva del cultivo de alfalfa se explicó de manera significativa por la triple interacción de las variables Propiedad-Maquinaria especializada-Riego tecnificado. Se presentó el máximo efecto cuando se es propietario del predio por el efecto conjunto de Maquinaria especializada-Riego tecnificado. La interacción Maquinaria especializada-Riego tecnificado muestra efecto significativo, pero su impacto fue menor cuando no se es propietario del predio.

\begin{abstract}
Introduction: The cultivation of alfalfa is the most used legume for feeding cattle worldwide due to its high nutritional value, mainly of protein, the District of Irrigation 05-Delicias is one of the most important producers of this crop in Mexico. The objective of this research was to determine the impacts of the different forms of use of factors of production on the productive efficiency, with the purpose of generating useful information for producers and authorities for decision making.
\end{abstract}

Method: In this study, 4 predictors, land tenure, property ownership, specialized machinery and technical irrigation were selected in dichotomous form, and a variable response, productive efficiency in alfalfa cultivation was used to determine the best fit of the model, using the method of variables selecting backward step, they were analyzed under the logistic regression model by CATMOD of the SAS 9.4 package using contrasts to determine effects of the variables on significant interactions.

Results: Significant differences were found in the three interaction: property-machinerytechnical irrigation, as well as individually in the individual variables specialized machinery and technical irrigation. By contrasting were determines the different effects of the variables within the triple interaction, standing out the potential impact of the technical irrigation-machinery 
combined, when the property is counted with a odds rate of 16 , followed by the effect of the machinery when the producers have the property with a odds rate of 13.56 and the effect of technical irrigation, when likewise the producers are owners of their land too with a rate of odds of 8.0 .

Discussion or Conclusion: The efficiency of alfalfa cultivation from the productive point of view showed that it is significantly impacted by the triple interaction of the variables propertymachinery-technical irrigation presenting the maximum effects when it has the property of the farm inside of that triple interaction by the combined effect of technical irrigation-machinery, likewise the technical-irrigated machinery-irrigation interaction shows significance, but its impact is less when the property is not owned.

\section{Introducción}

La alfalfa (Medicago sativa L.) es la leguminosa más utilizada a nivel mundial para la alimentación del ganado, su importancia se debe a la cantidad de forraje producido por unidad de superficie y a su alto valor nutritivo, principalmente por su contenido de proteína (Ríos et al, 2015; Vázquez-Vázquez et al., 2010; Rojas et al, 2016). El cultivo de la alfalfa mejora la calidad de los suelos al asociarse en sus raíces con bacterias fijadoras del nitrógeno, mejora la productividad en entornos de sequía y, en un esquema de rotación de cultivos, reduce erosión del suelo y mejora sus propiedades físicas (Moreira y Kumar, 2010). El cultivo demanda climas secos con altas temperaturas en verano, por lo que se le encuentra principalmente en regiones áridas y semiáridas (Rojas et al., 2016) donde el ambiente induce diferentes grados de estrés hídrico y los materiales utilizados tienen diferentes atributos para tolerar este estrés (Ray et al., 2015), como ocurre en el Distrito de Riego 05-Delicias (DR-05), en el Estado de Chihuahua.

A nivel mundial, se cultivan 30 millones de ha de alfalfa, teniendo América y Europa el $90 \%$ de la producción. En México, la superficie cultivada se ha mantenido constante en los últimos 10 años, fluctuando entre 380,000 y 390,000 ha, de las cuales el Estado de Chihuahua es el principal productor, con un $22 \%$ de la superficie total establecida (SIAP-SAGARPA, 2016). Dentro de los Distritos de Riego del país, en el ciclo agrícola 2014-2015 se cosecharon 142,312 ha de alfalfa, de las cuales 25,536 ha fueron en el DR-05, representando un 18\% de la superficie total de los Distritos de Riego del país (SEMARNAT, 2016). 
El DR-05 ha tenido un incremento gradual en la superficie cultivada de alfalfa, reportándose en el ciclo agrícola 1989-1990 un total de 11,914 ha, en 2004-2005 fueron 12,426 ha y en el 2014-2015 con 25,536 ha, representando 14.29, 39.00 y $41.62 \%$ de la superficie total cultivada respectivamente en esos ciclos, con un incremento del $214.34 \%$ para este periodo. En el ciclo agrícola 2015-2016, este cultivo ocupó casi la mitad (47\%) de la superficie total establecida en el DR-05. No obstante, que el DR-05 ha alcanzado una especialización en el cultivo de alfalfa, la eficiencia productiva presenta mucha variación en sus rendimientos en forma achicalada (heno), la más común en el DR-05, de 14.71 hasta 32.67 t ha-1, y en materia seca (MS) de 13.24 hasta 29.40 t ha-1, con promedios en rendimiento y MS de 17.98 y 16.17 t ha-1 respectivamente (SAGARPA-SIAP, 2016). Esta variabilidad se debe a las diferentes formas productivas y tipo de productor, por lo que para analizar esta situación se determinó tomar como variables explicatorias dicotómicas: Riego tecnificado, Maquinaria especializada, Propiedad del predio, y Régimen de tenencia de la tierra, permitiendo definir el impacto potencial de cada una de ellas y de sus interacciones sobre la eficiencia productiva en este cultivo.

Ávila et al. (2000) mencionaron que es fundamental distinguir las diferentes formas y procesos productivos que existen en una determinada área de estudio y sus resultados. El alto grado de heterogeneidad que existe entre las diversas formas de explotación en una región, dificulta la toma de decisiones. Sin embargo, al agrupar las explotaciones de acuerdo con sus principales diferencias y similitudes se logra homogenizar los productores en grupos y comprender mejor la heterogeneidad entre ellos y su impacto en la productividad (Cabrera et al., 2004).

Aravena y Hofman (2014) mencionaron que la productividad es el resultado de las decisiones acerca de la elección de cantidad y calidad de insumos productivos y de la tecnología empleada, de cómo cambian todos estos elementos y que un uso no adecuado de los recursos repercute en un decremento productivo y en un deterioro ambiental. INEGI (2014) determinó que en 2012 el costo por degradación y agotamiento de los recursos naturales en el país, fue de 1.7 y $4.6 \%$ respectivamente, representando un costo total del $6.3 \%$ del PIB nacional.

El uso eficiente de los recursos es fundamental para garantizar la producción alimentaria, la economía y el trabajo de las familias vinculadas con el sector agrícola. Se debe incrementar la eficiencia productiva, entendiéndose como la relación existente entre la producción de un cultivo por unidad de insumo (Salazar-Moreno et al., 2014). Se deben buscar estrategias para elevar los 
índices de productividad, es decir producir más con menos (CONAGUA, 2014). Además, se debe hacer énfasis en que este aumento en la productividad debe de ir relacionado con el uso sustentable de los recursos (CONAGUA, 2001).

El análisis de la eficiencia productiva genera información de utilidad para tomadores de decisiones y productores. El conocimiento de la problemática del DR-05 en el uso adecuado de los recursos permitirá hacer cambios estructurales que impliquen una actividad agrícola económicamente eficiente, socialmente equitativa y ambientalmente sustentable, promoviendo el desarrollo y la calidad de vida de la población (Oswald-Spring, 2011).

La problemática del DR-05 se ha analizado bajo perspectivas de variables técnicasproductivas principalmente del uso y manejo del recurso agua, pero no existen estudios que determinen una caracterización en conjunto de variables productivas y su impacto en la eficiencia en el cultivo de alfalfa. El presente trabajo se desarrolló como una alternativa metodológica para analizar las eficiencias en términos de uso de los recursos, con técnica estadística de datos categóricos mediante un modelo de regresión logística. Este tipo de modelos es una generalización del modelo de regresión lineal clásico para variables dependientes categóricas dicotómicas (Ato et al. 2013). Tiene la ventaja de no requerir normalidad multivariable y el de homocedasticidad (igualdad de varianzas), que son difíciles de verificar. Otra ventaja radica en su similitud con la regresión múltiple (Alderete, 2006).

La regresión logística estima valores de tasa de momios (TM) o razón de posibilidades (odds ratio en inglés). La TM representa una estimación del efecto potencial de las variables explicatorias sobre la variable respuesta, cuando este no puede estimarse directamente, estima en la variable dependiente, la probabilidad $\pi$ de obtener la respuesta de interés, en base a los valores de las variables independientes mediante un modelo específico (Irala, 1997).

Con base en lo anterior el objetivo del presente trabajo fue determinar el impacto potencial de las variables descritas en base a tipo de productor y del uso de los recursos productivos sobre la eficiencia productiva del cultivo de la alfalfa en el DR-05 utilizando análisis estadístico de datos categóricos con un modelo de regresión logística. 


\section{Materiales y métodos}

\section{Descripción del área de estudio}

El Distrito de Riego 05 (DR-05) ubicado en Cd. Delicias, Chihuahua, México, pertenece a la cuenca hidrológica del Río Bravo, se ubica en el norte de México, región centro sur del Estado de Chihuahua a $28^{\circ} 11^{\prime \prime}$ de latitud norte y a $105^{\circ} 28^{\prime}$ ' de longitud oeste, con una altitud media de 1,165 msnm. El DR-05 cuenta con una superficie de 100,093 ha (80\% de riego y $20 \%$ de temporal) con 8,113 productores (43\% ejidatarios y $57 \%$ pequeños productores).

\section{Sujeto de estudio}

La población objeto de estudio estuvo conformada por 2,432 productores de alfalfa del Distrito de Riego, con una superficie de 25,536 ha (ciclo agrícola 2014-2015).

\section{Tipo de investigación}

Esta investigación fue de tipo exploratoria, descriptiva y conclusiva. Exploratoria debido a que comprendió un tema no abordado con anterioridad en el DR-05, por lo que sus resultados, constituyen una visión aproximada del objeto de investigación. Descriptiva porque determina propiedades, características y perfiles importantes del proceso analizado. Conclusiva porque su objetivo es ayudar a la toma de decisiones manifestando conclusiones de los procesos mediante el análisis de la información.

\section{Elección del cultivo bajo estudio}

Para recabar la información de la producción agrícola, se realizaron entrevistas personales con las autoridades de CONAGUA, Jefe de Distrito y Jefe de Operación. Para delimitar el cultivo de estudio, de acuerdo a su importancia, se analizó información estadística de la producción agrícola proveniente de 14 ciclos, mediante el acceso a diferentes bases de datos (SEMARNAT, 2010; SEMARNAT, 2011; SEMARNAT, 2012; SEMARNAT, 2013).

\section{Determinación de la muestra}

Se aplicó una encuesta a 244 productores de alfalfa del DR-05 de Delicias, Chihuahua. México, que fueron seleccionados completamente al azar. Este tamaño de muestra asegura la representatividad de los productores de alfalfa del Distrito, con un $95 \%$ de confiabilidad y $5 \%$ de 
error de precisión, bajo el supuesto de varianza máxima. La encuesta fue diseñada con preguntas cerradas de respuesta dicotómica con base en las variables de interés sobre el proceso productivo del cultivo de alfalfa.

\section{Tipo de variables}

Se consideraron como variables explicativas: Tipo de productor (TENENCI), Propiedad del predio (PROP), Maquinaria especializada (MAQ), Riego tecnificado (RIEGOTE) y como variable de respuesta la Eficiencia productiva (EFIC) superior en un 20\% a la media del DR-05, 18.41 t de M.S. ha-1 ciclo-1, (SAGARPA-SIAP, 2016). El procesamiento de la información de las encuestas se realizó con el programa Excel de Microsoft Office (Office, 2013).

\begin{tabular}{llc}
\hline Variable & Descripción & Unidades \\
\hline Tenencia (TENENCI) & Pequeño propietario (PP) o ejidatario (E). & PP/E \\
Propiedad del predio (PROP) & Dueño (P) o arrendatario (p). & $\mathrm{P} / \mathrm{p}$ \\
$\begin{array}{l}\text { Maquinaria especializada } \\
\text { (MAQ) }\end{array}$ & $\begin{array}{l}\text { Cuenta con el equipo completo (M) o no posee el equipo } \\
\text { completo (m). }\end{array}$ & $\mathrm{M} / \mathrm{m}$ \\
$\begin{array}{l}\text { Riego tecnificado } \\
\text { (RIEGOTE) }\end{array}$ & Riego tecnificado (R) o riego tradicional por gravedad (r). & $\mathrm{R} / \mathrm{r}$ \\
Eficiencia productiva (EFIC) & $\begin{array}{l}\text { Rendimiento de materia seca en alfalfa por ciclo superior a } \\
22.04 \text { t ha-1 }\end{array}$ & $\mathrm{Si} / \mathrm{No}$ \\
\end{tabular}

\section{Análisis estadístico}

Se realizó, en primera instancia, un análisis estadístico descriptivo de las frecuencias de las variables. El análisis estadístico inferencial posterior utilizó un modelo de regresión logística mediante el procedimiento CATMOD del paquete SAS 9.4 (SAS, 2012), considerando las variables categóricas descritas como explicativas y la eficiencia productiva como variable de respuesta.

El modelo de regresión logística puede ser representado de la siguiente manera:

$$
\log \left(\frac{\pi}{1-\pi}\right)=\alpha+\beta i+\beta j+\beta k \ldots \beta z+\beta i j+\beta i k+\beta j k+\beta i j k \ldots \beta x y z
$$


Donde:

$\pi=\mathrm{P}(\mathrm{Y}=1 \mid \mathrm{x})$, es la probabilidad de que la variable respuesta $(\mathrm{Y})$ tome el valor de 1 dada una matriz de valores $\mathrm{X}$;

$\alpha, \beta \mathrm{i}, \beta \mathrm{j}, \beta \mathrm{k}, \beta \mathrm{ij} \ldots \beta \mathrm{z}+\beta \mathrm{ij}+\beta \mathrm{ik}+\beta \mathrm{jk}+\beta \mathrm{ijk} \ldots . . \beta \mathrm{xyz}$ son parámetros desconocidos, y X1...Xn son las variables explicativas (Agresti, 2002).

Para seleccionar las variables explicativas que resultaban en un mejor ajuste del modelo, se recurrió al método de selección de variables por pasos hacia atrás (Agresti, 2002).

Para analizar el efecto de interacción, se utilizaron contrastes analizándose la tasa de momios. La regresión logística utilizada para este propósito se expresa de la siguiente manera:

$$
\log \left(\frac{\pi 1 / \mathrm{ijk}}{\pi 2 / \mathrm{ijk}}\right)=\alpha+\beta \mathrm{i}+\beta \mathrm{j}+\beta \mathrm{k}+\beta \mathrm{ij}+\beta \mathrm{ik}+\beta \mathrm{jk}+\beta \mathrm{ijk}
$$

Donde: $\log \left(\frac{\pi 1 / \mathrm{ijk}}{\pi 2 / \mathrm{ijk}}\right)$ representa la tasa de momios, $\alpha$ es la constante del modelo, $\beta \mathrm{i}, \beta \mathrm{j}, \beta \mathrm{k}, \beta \mathrm{ij}$, $\beta \mathrm{ik}, \beta \mathrm{jk}$ y $\beta \mathrm{ijk}$ son parámetros desconocidos, y X1...Xn son las variables explicativas.

\section{Resultados y discusión}

\section{Caracterización de los agricultores y de los factores de productivos}

El uso de tablas dinámicas en Excel permitió el análisis descriptivo de las frecuencias de las variables explicativas y el uso de CATMOD de SAS permitió detectar el efecto de los factores de producción sobre la competitividad como variable de respuesta, resaltando el efecto de la interacción de tres factores $(\mathrm{p}=0.0203)$. La Figura 1 agrupa las combinaciones de los factores productivos con que cuentan los productores de alfalfa en el DR-05 de Delicias.

Al considerar los factores de forma general, se encontró que el riego tecnificado es utilizado sólo por el $25 \%$ de los productores, mientras que el resto siguen utilizando riego tradicional (por gravedad); un $61.5 \%$ de los productores cuentan con la maquinaria especializada para su proceso productivo y el $70 \%$ son dueños de los predios de alfalfa que cultivan.

Al analizar simultáneamente dos factores de producción, se encontró que sólo el $15.16 \%$ de los productores del DR-05 cuentan a la vez con Maquinaria especializada y Riego tecnificado, mientras que el $46.31 \%$ cuentan con Maquinaria especializada pero realizan el riego en forma 
tradicional (por gravedad); el $9.84 \%$ tienen Riego tecnificado pero no cuentan con Maquinaria especializada y cultivan en los lotes que usufructúan; y el $28.69 \%$ de los productores no cuentan ni con Maquinaria especializada ni con Riego tecnificado, esto debido a que algunos productores que rentan tierras procuran que les maquilen las labores y evitan, de esta manera, la inversión en capital fijo.

Al analizar conjuntamente los tres factores de producción se encontró que solo el $9.4 \%$ de los productores de alfalfa del DR-05 son Propietarios del predio que cultivan, poseen Maquinaria especializada y tienen Riego tecnificado (denotados PMR), lo que contrasta con el $8.6 \%$ renta la tierra que cultiva y no tiene Maquinaria especializada ni Riego tecnificado (denotados por pmr). El mayor porcentaje, $33.40 \%$, corresponde a los productores de alfalfa que son Propietarios del predio que cultivan, poseen Maquinaria especializada, pero utilizan riego por gravedad (PMr).

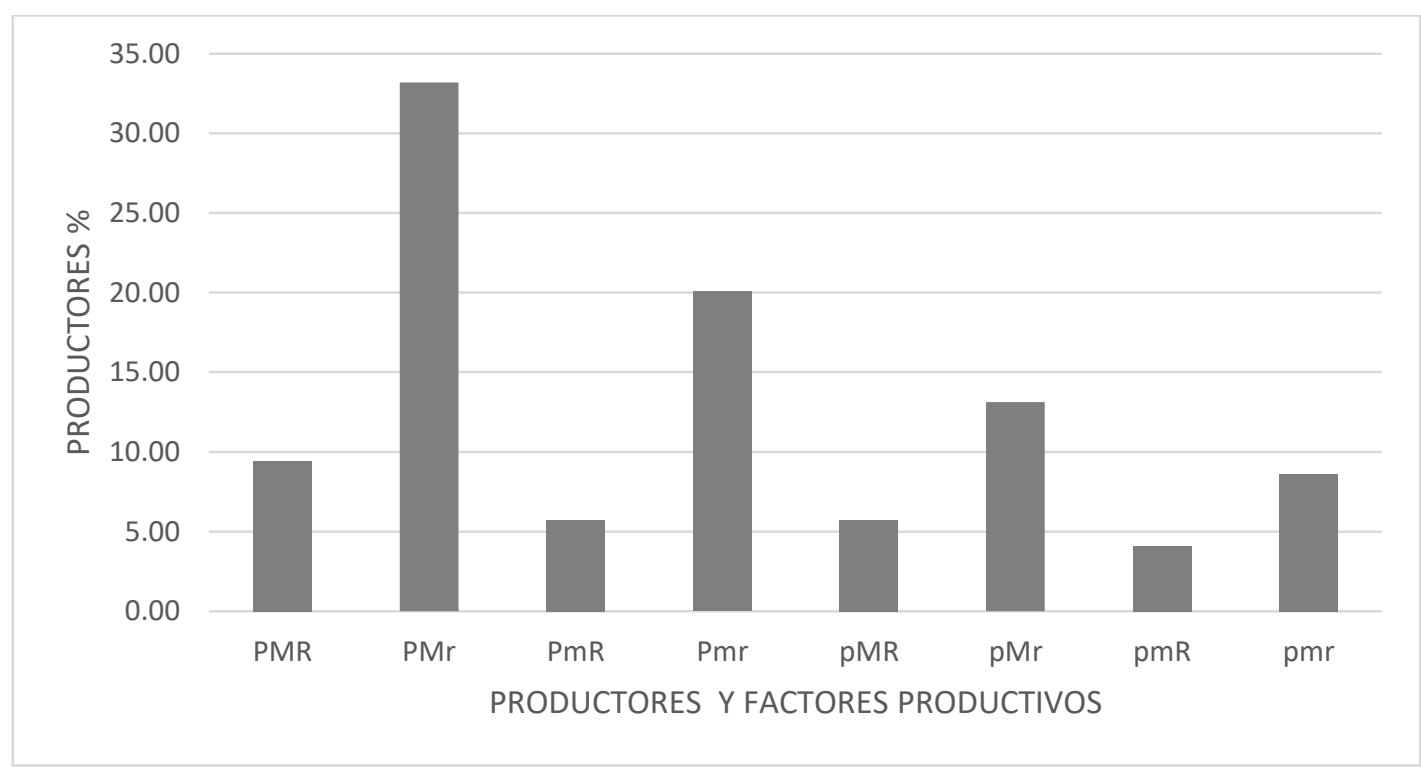

Figura 1. Combinación de los niveles de factores de producción y sus frecuencias en productores de alfalfa en el Distrito de Riego 05-Delicias.

Las literales mayúsculas indican que el productor posee el factor de producción específico $(\mathrm{P}=$ Propiedad de la tierra, $\mathrm{M}=$ Maquinaria especializada y $\mathrm{R}=$ Riego tecnificado); mientras que las letras minúsculas indican que no se posee ese factor ( $\mathrm{p}, \mathrm{m}$ y $\mathrm{r}$, respectivamente). 


\section{Eficiencia productiva}

La producción en el DR-05 presentó gran variación, con valores de materia seca que oscilan desde 13.24 hasta 29.40 t ha-1 por ciclo agrícola. Esta variación puede explicarse por diferentes condiciones en los factores productivos, tales como el uso de Maquinaria, Sistema de riego, etc.

La Propiedad no afectó la productividad de manera general (Cuadro 1); mientras que la Maquinaria especializada y el Riego tecnificado mostraron efecto en lo general $(\mathrm{p}=0.0010)$, aunque este efecto varió dependiendo con qué nivel de los otros dos factores se combinaban (efecto significativo de la triple interacción, con $\mathrm{p}=0.0203$ ).

Cuadro 1. Variables del modelo completo y su efecto.

\begin{tabular}{|c|c|c|c|c|c|}
\hline Parámetro & & Estimador & $\begin{array}{l}\text { Error } \\
\text { estándar }\end{array}$ & $\begin{array}{l}\text { Chi- } \\
\text { cuadrado }\end{array}$ & $\begin{array}{l}\text { Pr > } \\
\text { ChiSq }\end{array}$ \\
\hline Término independiente & & -0.2124 & 0.1971 & 1.16 & 0.2812 \\
\hline TENENCI & EJ & 0.2818 & 0.2009 & 1.97 & 0.1607 \\
\hline PROP & $\mathrm{N}$ & 0.1403 & 0.1964 & 0.51 & 0.4750 \\
\hline MAQ & $\mathrm{N}$ & 0.7555 & 0.1988 & 14.44 & 0.0001 \\
\hline RIEGOTE & $\mathrm{N}$ & 0.6355 & 0.1929 & 10.85 & 0.0010 \\
\hline TENENCI*PROP & EJ N & -0.0389 & 0.1720 & 0.05 & 0.8209 \\
\hline TENENCI*MAQ & EJ N & 0.2221 & 0.1795 & 1.53 & 0.2159 \\
\hline TENENCI*RIEGOTE & EJ N & -0.1581 & 0.1869 & 0.72 & 0.3978 \\
\hline PROP*MAQ & $\mathrm{N} \mathrm{N}$ & -0.0782 & 0.1916 & 0.17 & 0.6833 \\
\hline PROP*RIEGOTE & $\mathrm{NN}$ & 0.0367 & 0.1875 & 0.04 & 0.8446 \\
\hline MAQ*RIEGOTE & $\mathrm{NN}$ & 0.1063 & 0.1896 & 0.31 & 0.5751 \\
\hline PROP*MAQ*RIEGOTE & $\mathrm{NNN}$ & -0.4360 & 0.1879 & 5.38 & 0.0203 \\
\hline
\end{tabular}

TENENCI $=$ Régimen de tenencia de la tierra, $\mathrm{PROP}=$ Propiedad de la tierra, MAQ= Maquinaria especializada y RIEGOTE $=$ Riego tecnificado. 
El análisis de regresión logística permitió elegir el siguiente modelo, como el modelo que explicó mejor la relación entre las variables predictoras originales y la eficiencia productiva como variable de respuesta:

$$
\begin{aligned}
& \log (\pi / 1-\pi)=-.2924+.0806 \mathrm{PROP}+.6917 \mathrm{MAQ}+.6227 \mathrm{RIEGOTE}-.1333 \mathrm{PROP} * \mathrm{MAQ} \\
& +.0614 \text { PROP*RIEGOTE + .0705MAQ*RIEGOTE }-.4079 \mathrm{PROP} * \mathrm{MAQ} * \mathrm{RIEGOTE}
\end{aligned}
$$

Donde: $\pi=\mathrm{P}(\mathrm{Y}=1 \mid \mathrm{x})$, es la probabilidad de que la variable respuesta $(\mathrm{Y})$ tome el valor de 1 dada una matriz de valores $\mathrm{X}$, TENENCI= Régimen de tenencia de la tierra, PROP= Propiedad de la tierra, MAQ= Maquinaria especializada y RIEGOTE= Riego tecnificado.

La triple interacción resultó significativa (Cuadro 2), por lo que se analizó el efecto de sus componentes (Cuadro 2).

Cuadro 2. Variables del modelo seleccionado y su efecto.

\begin{tabular}{llllll}
\hline Parámetro & & Estimador & $\begin{array}{l}\text { Error } \\
\text { estándar }\end{array}$ & $\begin{array}{l}\text { Chi- } \\
\text { cuadrado }\end{array}$ & Pr $>$ ChiSq \\
\hline Término independiente & & -0.2924 & 0.1809 & 2.61 & 0.1059 \\
PROP & $\mathrm{N}$ & 0.0806 & 0.1809 & 0.20 & 0.6558 \\
MAQ & $\mathrm{N}$ & 0.6917 & 0.1809 & 14.63 & 0.0001 \\
RIEGOTE & $\mathrm{N}$ & 0.6227 & 0.1809 & 11.85 & 0.0006 \\
PROP*MAQ & $\mathrm{N} \mathrm{N}$ & -0.1333 & 0.1809 & 0.54 & 0.4611 \\
PROP*RIEGOTE & $\mathrm{N} \mathrm{N}$ & 0.0614 & 0.1809 & 0.12 & 0.7344 \\
MAQ*RIEGOTE & $\mathrm{N} \mathrm{N}$ & 0.0705 & 0.1809 & 0.15 & 0.6968 \\
PROP*MAQ*RIEGOTE & $\mathrm{N} \mathrm{N} \mathrm{N}$ & -0.4079 & 0.1809 & 5.09 & 0.0241 \\
& & & & & \\
\hline
\end{tabular}

PROP $=$ Propiedad de la tierra, MAQ $=$ Maquinaria especializada y RIEGOTE $=$ Riego tecnificado. 
De esta manera se determinaron, considerando esta triple interacción, los diferentes efectos potenciales sobre la eficiencia productiva (Cuadro 3).

Cuadro 3. Prueba de Contraste de estimadores de verosimilitud máxima (tasa de momios) y su significancia.

\begin{tabular}{lllllll}
\hline $\begin{array}{l}\text { Contraste triple } \\
\text { vs triple }\end{array}$ & Estimador & Error & $\begin{array}{l}\text { Chi- } \\
\text { cuadrado }\end{array}$ & Pr $>$ ChiSq & Límites de confianza \\
& & Estándar & & & \\
\hline pmr vs pMr & 1.5556 & 0.9087 & 0.57 & 0.4495 & 0.4950 & 4.8881 \\
pmR vs pMR & 6.0000 & 5.9491 & 3.27 & 0.0707 & 0.8594 & 41.8920 \\
Pmr vs PMr & 13.5556 & 6.1000 & 33.56 & $<.0001$ & 5.6115 & 32.7459 \\
PmR vs PMR & 2.0000 & 1.5055 & 0.85 & 0.3572 & 0.4574 & 8.7456 \\
pmr vs pmR & 2.0000 & 1.5675 & 0.78 & 0.3765 & 0.4304 & 9.2933 \\
pMr vs pMR & 7.7143 & 6.5006 & 5.88 & 0.0153 & 1.4792 & 40.2324 \\
Pmr vs PmR & 8.0000 & 5.3499 & 9.67 & 0.0019 & 2.1570 & 29.6705 \\
PMr vs PMR & 1.1803 & 0.6697 & 0.09 & 0.7701 & 0.3882 & 3.5890 \\
pmr vs Pmr & 0.4500 & 0.2664 & 1.82 & 0.1773 & 0.1410 & 1.4357 \\
pmR vs PmR & 1.8000 & 1.5179 & 0.49 & 0.4858 & 0.3447 & 9.3987 \\
pMr vs PMr & 3.9214 & 1.7244 & 9.66 & 0.0019 & 1.6563 & 9.2845 \\
pMR vs PMR & 0.6000 & 0.5495 & 0.31 & 0.577 & 0.0997 & 3.6113 \\
Pmr vs PMR & 16.0000 & 10.0132 & 19.63 & $<.0001$ & 4.6926 & 54.5538 \\
\hline
\end{tabular}

Las literales mayúsculas indican que el productor posee el factor de producción específico (P = Propiedad de la tierra, $\mathrm{M}=$ Maquinaria especializada y $\mathrm{R}=\mathrm{Riego}$ tecnificado); mientras que las letras minúsculas indican que no se posee ese factor (p, $\mathrm{m}$ y r, respectivamente).

\section{Maquinaria (MAQ)}

En general, si un productor de alfalfa del DR-05 cuenta o no con Maquinaria especializada, afecta la eficiencia productiva ( $\mathrm{p}=0.0006)$; sin embargo, este efecto varió de acuerdo con los niveles de Propiedad (propietario o renta) y tipo de Riego (tecnificado o por gravedad) con que se combinó. En este sentido, las pruebas de contrastes indican que los productores de alfalfa del DR-05 que son propietarios del predio que cultivan y utilizan riego por gravedad, aumentan 13.56 veces (Tasa de momios) la probabilidad de lograr la eficiencia productiva en su predio $(\mathrm{p}<0.0001)$ por efecto del uso de Maquinaria especializada (Pmr vs PMr). 


\section{Riego tecnificado (RIEGOTE)}

El Riego tecnificado mostró efecto significativo en lo general ( $\mathrm{p}=0.0006)$ sobre la productividad del cultivo de alfalfa; sin embargo, por efecto de interacción se detectó que este efecto varió de acuerdo a los niveles de Propiedad y tipo de Maquinaria con que se combinó. De esta manera, al analizar las pruebas de contrastes, se encontró que los productores de alfalfa del DR-05 que son propietarios del predio que cultivan, pero que no cuentan con Maquinaria especializada, aumentan 8 veces la probabilidad de lograr la eficiencia productiva $(\mathrm{p}=0.0019)$ al usar Riego tecnificado en vez de riego por gravedad (Pmr vs PmR).

Por otro lado, los productores que rentan el predio que cultivan y cuentan con Maquinaria especializada, aumentan 7.7 veces la probabilidad de lograr la eficiencia productiva $(\mathrm{p}=0.0153)$ por efecto del uso de Riego tecnificado en sustitución del riego por gravedad (pMr vs pMR).

\section{Propiedad (PROP)}

En general, si un productor de alfalfa del DR-05 es dueño o renta el predio que siembra, no afecta la eficiencia productiva $(\mathrm{p}=0.6558)$; sin embargo, cuando un productor cuenta con Maquinaria especializada y no se tiene Riego tecnificado ( $\mathrm{pMr}$ vs $\mathrm{PMr}$ ), ser propietario del predio incrementa 3.92 veces la probabilidad de lograr la eficiencia productiva $(\mathrm{p}=0.0019)$.

\section{Maquinaria-Riego tecnificado (MAQ-RIEGOTE)}

La combinación de factores productivos que mostró el mayor impacto potencial $(\mathrm{p}<0.0001)$ sobre la eficiencia productiva de la alfalfa en el DR-05 de Delicias, ocurrió dentro de los productores que son dueños de su predio, donde, cuando el productor cuenta Maquinaria especializada y Riego tecnificado (PMR) incrementa 16 veces la probabilidad de lograr la eficiencia productiva, frente a los propietarios de su predio pero que no tienen ni Maquinaria especializada ni Riego tecnificado.

En general, el Cuadro 3 muestra valores altos de los errores estándar de los estimadores y por lo tanto intervalos de confianza amplios para los mismos, situación que se genera porque en el fenómeno de la eficiencia productiva del cultivo de alfalfa están frecuentemente implícitos una gran cantidad de variables adicionales a las aquí estudiadas (ambientales, sociales y económicos)

y que al ser este un muestreo bajo las condiciones reales en que se da la producción de este cultivo, no se controla experimentalmente esas variables. 
Estos resultados concuerdan con los trabajos llevados a cabo por investigadores como Ortega-Gaucin et al. (2009) que indicaron que la tecnificación del riego en el DR-05 logrará incrementar la eficiencia y obtener más disponibilidad de agua, permitiendo una mayor superficie cosechada, diversificando la composición del patrón de cultivos, lo cual incidió directamente en un mayor beneficio neto.

En este sentido se coincide con Dzul et al. (2011) quienes mencionaron que una de las soluciones planteadas para mejorar la eficiencia de producción es la tecnificación del riego, pues un mal uso del agua repercute en una producción ineficiente. De igual manera Olvera et al. (2014) sustentaron que la tecnificación del riego, mediante acciones de rehabilitación y modernización de la infraestructura hidráulica concesionada en los Distritos de Riego, permite una mayor eficiencia, mejorando la calidad e incrementar la producción.

Sánchez-Hernández et al. (2014) mencionaron la necesidad de implementar programas de apoyo con maquinaria adecuada a las necesidades específicas de producción agrícola local, propiciando una mayor eficiencia productiva. Estos mismos autores indican que la caracterización de la producción es de suma importancia como herramienta para la toma de decisiones sobre la operación y orientación de futuros programas de apoyo gubernamental.

En general, este trabajo concuerda con las bondades señaladas por los factores de producción estudiados y reportados en la literatura citada, pero el aporte sustancial del presente trabajo es que se analiza y determina específicamente los impactos de cada variable y sus interacciones.

Estos resultados representan una herramienta para un uso más adecuado de los recursos de producción, buscando la productividad a través de una tecnificación del riego y/o mecanización especializada que consideren el tipo de productores que aquí se indican y pudiendo lograr incrementos potenciales en los rendimientos, como los señalados por Olvera et al. (2014), de un 30 a un $60 \%$.

\section{Conclusiones}

El modelo de regresión logística que mejor explicó el comportamiento de la eficiencia productiva del cultivo de alfalfa en el DR-05 incluyó a los factores de producción de Propiedad del predio, Maquinaria especializada y Riego tecnificado. De manera global, el uso de Maquinaria especializada y el Riego tecnificado mostraron los mayores efectos; sin embargo, al ser la triple 
interacción significativa, el efecto de estos factores varió dependiendo de con que niveles de los otros factores se combinarán.

El impacto potencial de mayor magnitud ocurre dentro de los productores que son dueños del predio que cultivan, pues estos incrementan 16 veces la probabilidad de lograr la eficiencia productiva cuando cuentan con Maquinaria especializada y Riego tecnificado frente a la situación en que no se cuenta con estos dos factores de producción.

Los productores que son Propietarios del predio que cultivan y utilizan Riego por gravedad, aumentan 13.56 veces la probabilidad de lograr la eficiencia productiva en su predio el uso de Maquinaria especializada.

Los productores que son propietarios del predio que cultivan, pero que no cuentan con Maquinaria especializada, aumentan 8 veces la probabilidad de lograr la eficiencia productiva al usar Riego tecnificado en vez de riego por gravedad.

La información derivada de este estudio sirve como base para la toma de decisiones de productores, investigadores y autoridades con injerencia en el DR-05 Delicias, puesto que, aunque la importancia de los factores de producción aquí estudiados ya se ha señalado en trabajos anteriores, este trabajo cuantifica los efectos potenciales de cada factor de producción en situaciones de interacción específicas de niveles de los factores.

\section{$\underline{\text { Referencias }}$}

Agresti A. (2002). Categorical data analysis. Second edition. New York, NY: John Wiley \& Sons; 2002.

Alderete, AM. (2006). Fundamentos del análisis de regresión logística en la investigación psicológica. Revista Evaluar, 6 (2006): 52-67.

Aravena, C., y Hofman, AA. (2014). Crecimiento económico y productividad en América Latina: Una perspectiva por industria, según la base de datos LA-KLEMS. Naciones unidas, CEPAL, Serie Macroeconomía del Desarrollo N 152 , Santiago de Chile

Ato, M., López, JJ., Benavente, A. (2013). Un sistema de clasificación de los diseños de investigación en psicología. Revista Anales de psicología, 29(3): 1038-1059.

Ávila, L., M. Muños y B. Rivera. 2000. Tipificación de los sistemas de producción agropecuaria en la zona de influencia del programa UNIR (CALDAS). Análisis de Conglomerados y 
Componentes Principales. Universidad de Caldas, Departamento de sistemas de producción. Programa UNIR. 231 pp.

Cabrera, D., García, A., Acero, R., Castaldo, A., Perea, J., Martos, J. (2004). Metodología para la caracterización y tipificación de sistemas ganaderos. Universidad de Córdoba Documentos de trabajo producción animal y gestión.

Comisión Nacional del Agua (CONAGUA). (2001). Programa Nacional Hidráulico 2001-2006. Gobierno federal. México.

Comisión Nacional del Agua (CONAGUA). (2014). Estadísticas Agrícolas de los Distritos de Riego Año Agrícola 2012-2013. Tlalpan, D.F. México.

Dzul, CT., Ramírez Jaramillo, G., Sánchez Cohen, I., Lomas Barrié, CT., Cano González, ADJ. (2011). Diagnóstico y evaluación de sistemas de riego en el distrito 048 Ticul, Yucatán. Revista mexicana de ciencias agrícolas, 2: 5-18.

Instituto Nacional de Estadística, Geografía e Informática (INEGI). (2014). Sistema de cuentas económicas y ecológicas de México, INEGI.2013. D.F. México.

Irala, JD., Fernández-Crehuet Navajas, R., Serrano del Castillo, A. (1997). Intervalos de confianza normalmente amplios en regresión logística: Interpretación de resultados de programas estadísticos. Revista Panam Salud Publica/Pan Am J Public Health, 1(3): 230234.

Olvera Salgado, MD., Ojeda Bustamante, W., Bahena Delgado, G., Alpuche Garcés, O. (2014). Participación y apropiación de la modernización y tecnificación del riego en Chihuahua México. Revista Ingeniería Hidráulica y Ambiental, 35(1): 47-61.

Ortega-Gaucin, D., Mejía Sáenz, E., Palacios Vélez, E., Rendón Pimentel, L., Exebio García, A. (2009). Modelo de optimización de recursos para un distrito de riego. Revista Terra Latinoamericana, 27(3): 219-226.

Oswald-Spring, U. (2011). Retos de la investigación del agua en México. Cuernavaca, México: Centro Regional Investigaciones Multidisciplinarias, UNAM, 754 pp.

Ray, IM., Han, Y., Meenach, CD., Santantonio, N., Sledge, MK., Pierce, CA., Sterling, TM., Kersey, RK., Bhandari HS., Monteros MJ. (2015). Identification of quantitative trait loci for alfalfa forage biomass productivity during drought stress. Crop Science, 55(5): 20122033. 
Ríos Flores, JL., Torres Moreno, M., Castro Franco, R., Torres Moreno, M. A., Ruiz Torres, J. (2015). Determinación de la huella hídrica azul en los cultivos forrajeros del DR-017, Comarca Lagunera, México. Revista de la Facultad de Ciencias Agrarias. Universidad Nacional de Cuyo, 47(1): 93-107.

Rojas García, AR., Hernández-Garay, A., Cansino, SJ., Maldonado Peralta, MDLA., Mendoza Pedroza, SI., Álvarez Vázquez, P., Joaquín Torres, BM. (2016). Comportamiento productivo de cinco variedades de alfalfa. Revista Mexicana de Ciencias Agrícolas, 7(8): $1855-1866$.

Salazar-Moreno, R., Rojano-Aguilar, A., López-Cruz, I. L. (2014). La eficiencia en el uso del agua en la agricultura controlada. Revista Tecnología y ciencias del agua, 5(2): 177-183.

Sánchez-Hernández, MA., Ayala-Garay, AV., Cervantes-Osornio, R., Garay-Hernández, M., la O-Olán, D., Martínez-Trejo, G., Velázquez-López, N. (2014). Diagnóstico de la maquinaria agrícola en Amecameca y Texcoco, Estado de México. Revista Agricultura, sociedad y desarrollo, 11(4): 499-516.

Secretaría de Agricultura, Ganadería, Pesca y Alimentación (SAGARPA). Sistema de información agroalimentaria y pesquera (SIAP). (2016) http://www.siap.sagarpa.gob.mx/

Secretaria del medio ambiente y recursos naturales (SEMARNAT). (2010). Estadísticas agrícolas de los distritos de riego Año agrícola 2008-2009. México.

Secretaria del medio ambiente y recursos naturales (SEMARNAT). (2011). Estadísticas agrícolas de los distritos de riego Año agrícola 2009-2010. México.

Secretaria del medio ambiente y recursos naturales (SEMARNAT). (2012). Estadísticas agrícolas de los distritos de riego Año agrícola 2010-2011. México.

Secretaria del medio ambiente y recursos naturales (SEMARNAT). (2013). Estadísticas agrícolas de los distritos de riego Año agrícola 2011-2012. México

Secretaria del medio ambiente y recursos naturales (SEMARNAT). (2016). Estadísticas agrícolas de los distritos de riego Año agrícola. México.

Vázquez-Vázquez, C., García-Hernández, JL., Salazar-Sosa, E., Murillo-Amador, B., OronaCastillo, I., Zúñiga-Tarango, R., Rueda-Puente, EO., Preciado-Rangel, P. (2010). Rendimiento y valor nutritivo de forraje de alfalfa (Medicago sativa L.) con diferentes dosis de estiércol bovino. Revista mexicana de ciencias pecuarias, 1(4): 363-372. 